\title{
Integrable Graded Manifolds and Nonlinear Equations
}

\author{
M. V. Saveliev
}

Institute for High Energy Physics, P.O. Box 35, Protvino, Serpukhov, SU-142284 Moscow Region, USSR

\begin{abstract}
A method is proposed for the classification of integrable embeddings of $(2+2)$-dimensional supermanifolds $V_{2 \mid 2}$ into an enveloping superspace supplied with the structure of a Lie superalgebra. The approach is first applied to the "even part" of the scheme, i.e. for the embeddings of 2-dimensional manifolds $V_{2}$ into Riemannian or non-Riemannian enveloping space. The general consideration is also illustrated by the example of superspaces supplied with the structure of the series sl $(n, n+1)$, whose integrable supermanifolds are described by supersymmetrical 2-dimensional Toda lattice type equations. In particular, for $n=1$ they are described by the supersymmetrical Liouville and Sine-Gordon equations.
\end{abstract}

1.

This paper is mainly devoted to a construction for classifying integrable embeddings of $(2+2)$-dimensional supermanifolds $V_{2 \mid 2}$ into the enveloping superspace $V_{N \mid M}$ supplied with the structure of a finite-dimensional Lie superalgebra $\quad \mathfrak{G}=\mathfrak{b}_{\overline{0}} \oplus \mathfrak{b}_{\overline{1}}$ (with the product [,]), whose $\mathbb{Z}$-grading $\left(\mathfrak{G}=\bigoplus_{m \in \mathbb{Z}} \mathfrak{G}_{m},\left[\mathfrak{G}_{m}, \mathfrak{G}_{n}\right] \subset \mathfrak{G}_{m+n}\right)$ is consistent with the $\mathbb{Z}_{2}$-grading, i.e. $\mathfrak{G}_{\overline{0}}=\oplus \mathfrak{G}_{2 m}$, $\mathfrak{G}_{\overline{1}}=\oplus \mathfrak{G}_{2 m+1}$.

Henceforth we use the following definitions. Denote

$\mathfrak{R}_{\mathfrak{H B}}(Z ; \mathfrak{I}) \equiv\left[\partial / \partial Z^{\mathfrak{A}}+\mathfrak{I}_{\mathscr{U}}(Z), \partial / \partial Z^{\mathfrak{B}}+\mathfrak{I}_{\mathfrak{B}}(Z)\right]$,

$1 \leqq \mathfrak{A}<\mathfrak{B} \leqq \mathfrak{N}+\mathfrak{M}$, where $\mathfrak{I}_{\mathfrak{A}} \equiv \sum_{1 \leqq \kappa \leqq \operatorname{dim} \mathfrak{F}} a_{\mathfrak{l}}^{\kappa}(Z) F_{\kappa}$ are some functions of $Z^{\mathfrak{A l}}$ taking values in the Grassmann hull $(\mathfrak{b}(\Lambda)$ of the superalgebra $(\mathfrak{5}$ with the basis $F_{\kappa}, \mathfrak{I}_{\mathscr{I}} \dot{\in}\left(\mathfrak{G}(\Lambda) ; Z^{A}=y^{A}, 1 \leqq A \leqq \mathfrak{N}\right.$, are usual Cartesian coordinates, $Z^{\Omega+\Re}=\Theta^{\Omega}$, $1 \leqq \Omega \leqq \mathfrak{M}$, are canonical generators of the Grassmann algebra $\Lambda_{\mathfrak{m}}$.

By a grading spectrum of $A_{ \pm}$we understand the choice of a $\mathbb{Z}$-grading of the superalgebra $\mathfrak{G}$ and the condition that for $\mathfrak{N}=2, \mathfrak{M}=0$ the operators $A_{ \pm}\left(z_{+}, z_{-}\right)$ 
$\equiv \mathfrak{I}_{1}(y) \pm i \mathfrak{I}_{2}(y), \quad z_{ \pm} \equiv \frac{1}{2}\left(y^{1} \mp i y^{2}\right)$, take the values in the subsuperspace $\bigoplus_{m \leq m_{ \pm}} \mathfrak{G}_{ \pm m}$ with some fixed numbers $m_{ \pm} \in \mathbb{Z}$. Here as an integrable sector of the

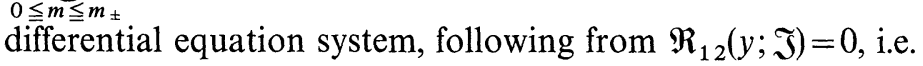

$$
\left[\partial / \partial z_{+}+A_{+}, \partial / \partial z_{-}+A_{-}\right]=0,
$$

$A_{ \pm} \dot{\epsilon} \underset{0 \leqq m \leqq m_{ \pm}}{\bigoplus_{ \pm m}} \mathfrak{G}_{ \pm m}$ one should understand a set of conditions for the functions $a_{ \pm}^{\kappa}\left(z_{+}, z_{-}\right)$, which make the given system exactly integrable. This means that the system is exactly integrable in the sense that it allows, under these conditions, a complete solution of the Cauchy problem, or will become completely integrable (in the sense of the inverse scattering problem) with the corresponding class of solitontype solutions.

Note representation (1.2) is form-invariant with respect to the gauge transformation

$$
A_{ \pm} \rightarrow \tilde{g}_{0}^{-1} A_{ \pm} \tilde{g}_{0}+\tilde{g}_{0}^{-1} \partial \tilde{g}_{0} / \partial z_{ \pm},
$$

which conserves the grading spectrum of the operators $A_{ \pm}$at $\tilde{g}_{0} \in \widetilde{G}_{0}$ due to the condition $\left[\mathfrak{G}_{0}, \mathfrak{b}_{m}\right] \subset \mathfrak{G}_{m}$. (Here $\tilde{g}_{0}$ is an element of the complex hull $\tilde{G}_{0}$ of the Lie group $G_{0}$ with the Lie algebra $\left(\mathfrak{5}_{0}\right.$.)

To clarify the approach developed and, moreover, the statement of the problem itself, we shall first discuss the "even part" of our construction announced in [1]. Namely, we consider, first of all, the embeddings of 2-dimensional manifolds into the conventional space (only with the Bose-type coordinates). This will allow us to perform the corresponding generalizations for supermanifolds without excessive concretization of the geometrical structures and, at the same time, without prejudice to understanding.

A classical problem of geometry is a classification of manifolds embedded in this or that fashion into enveloping space. According to the embedding theorems of differential geometry [2-5] a manifold $V_{n}$ is defined up to the motion in the enveloping space $V_{N}$ as a whole by its fundamental tensors satisfying the Gauss, Peterson-Codazzi, and Ricci equations (see Appendix A). Thus, one of the major aspects of the constructive solution of the problem involved consists in the investigation of these equations with a view to picking out their exactly and completely integrable subclasses, classifying the latter and finding the solutions. The characteristics of enveloping space (particularly its connections at the points of $V_{n}$ ) enter in the equations for the tensors of $V_{n}$ as "external" functions. For this reason it appears to be convenient to consider the triple of embedded manifolds $V_{n} \subset V_{N} \subset R_{M}$ for the self-consistent justification of the problem where the curvature tensor of $R_{M}$ has zero components.

From the technical point of view the problems encountered are concerned with the following. The equations under consideration represent a very complicated system of nonlinear partial differential equations with the arbitrary choice of the (pseudo-) normals to the manifold $V_{n}$ as well as the parameters on it. (In this connection it is also important to reformulate the equations in question in terms of the reduced functions, the latter possessing no arbitrariness mentioned above and serving for the gauge invariant quantities.) Apart from that, even with adequate methods for their solution, an approach is to be worked out to identify exactly or completely integrable subclasses of manifolds. 
Quite similar to the geometrical problem discussed is the fully solved problem of the classification of all semisimple subalgebras of simple Lie algebras. Thus it is natural to expect the application of the algebraic notions and methods in this or that form to be adequate to the solution of the embedding problem of differential geometry. Recently it was established [6,7] that the treatment of the classification problem of simple Lie algebras $(\mathfrak{5}$ and the description of the embeddings of threedimensional subalgebras in $\mathbf{6}$ are closely linked with the problem of picking out exactly and completely integrable two-dimensional nonlinear systems. This relationship serves as a hint to the possibility of a constructive solution of the differential geometry problem. (The concept in question in fact goes back to the pioneering idea conceived by $\mathrm{S}$. Lie to the effect that the continuous transformation groups have a decisive significance for the integration of partial differential equations and mean the same for the latter as the Galois groups for the algebraic equations.)

With the algebraic approach [6] to the construction of exactly and completely integrable nonlinear systems the starting point is a Lie algebra $(5$, the representation (1.2), and the choice of a grading spectrum of the operators $A_{ \pm}$in the integrable sector. At the same time differential geometry starts with the Gauss, Peterson-Codazzi, and Ricci equations themselves. Here the characteristics of $V_{N}$ (to be more exact, the structure of a Lie group with which $V_{N}$ is supplied) play the role of $(\mathfrak{b}$ introduction, while the choice of a grading spectrum provide the conditions for the components of the fundamental tensors of $V_{n}$. To reveal the geometrical implication of these conditions is of crucial importance; so are the notions of the grading spectrum.

The algebraic approach is quite applicable to the study of the embedding problem specifically for two-dimensional manifolds of differential geometry because up to now it failed to be extended to the systems in spaces with more than two dimensions. (The same is true for the other methods for integration of nonlinear equations.)

The construction proposed is based on the reformulation of the Gauss, Peterson-Codazzi, and Ricci equations for a manifold $V_{2}$ with the coordinates $y^{1}, y^{2}$ in the form of the Lax-type representation (1.2). Here the functions $a_{ \pm}^{\kappa}\left(z_{+}, z_{-}\right)$are expressed via the components of the basic fundamental tensors of $V_{2}$. Imposing definite conditions for the coefficients of the third fundamental form of $V_{2}$, we single out the so-called integrable class of the embeddings of $V_{2}$ into the flat space $R_{N}{ }^{1}$, which is described by the equations in an integrable sector. These conditions follow as a consequence of setting a grading spectrum of the operators $A_{ \pm}$. The choice of a grading spectrum allows us to select an integrable class of twodimensional nonlinear equations with the help of an algebraic approach [6], which makes it possible to obtain their exact solutions. In particular, consider the grading spectrum of the operators $A_{ \pm}$in the finite-dimensional simple Lie algebra $(\mathfrak{5}$ over $\mathbb{C}$, whose grading is consistent with an arbitrary integral embedding 3 of the three-dimensional subalgebra $\mathrm{sl}(2)$ in it $^{2}$ [9] and $m_{ \pm}=1$. Then each non-

1 The description of the embeddings of $V_{2}$ into the enveloping space $V_{N}$ of a non-zero curvature is provided by the consideration of the triple of spaces $V_{2} \subset V_{N} \subset R_{N+p}$ and the subsequent reduction [8]

2 Note that the Cartan element $H$ of $s 1(2)$ realizes the grading of $\mathfrak{5},\left[H, \mathfrak{b}_{m}\right]=2 m \mathfrak{5}_{m}$ 
equivalent $z$ is associated with the definite type of the integrable manifold $V_{2}$ (the minimal, constant curvature manifolds, and the intermediate variants including; for concrete examples see Appendix B) and it is described by the system of type (1.4) with $\xi_{ \pm}=0,\left(\mathfrak{5}=\mathfrak{G}_{\overline{0}}\right)$.

To realize this programme in the case of the supermanifolds $V_{2 / 2}$ it seems natural to try to describe their embeddings in the framework of a similar scheme. This means choosing a grading of the Lie superalgebra $(\mathfrak{b}$ (with which the enveloping superspace is supplied), consistent with the embeddings of the superalgebra $\mathrm{sl}(2)$ or $\operatorname{osp}(1,2)$, certainly if there is any in $\mathbf{6}$. Here, however, the equations following from the Lax-type representation (1.2) do not always turn out to be supersymmetric. (The latter is of an essential significance for physical applications, particularly, e.g. for the supersymmetric gauge and string approaches, and supergravity; see e.g. [10].)

Thus the construction proposed is as follows. We must formulate the problem for embedding supermanifolds $V_{2 \mid 2}$ as the representation (1.2) with the even elements $A_{ \pm}$taking values in the Grassmann hull $(\mathfrak{5}(\Lambda)$ of the superalgebra 65 , and also to set their grading spectrum. This leads to the superalgebraic Gauss, Peterson-Codazzi, and Ricci equations in an integrable sector. Here the evenness of $A_{ \pm}$ensures the possibility to apply the algebraic method for the integration of nonlinear partial differential equations [6]. Note, that in the framework of this method it is possible, in particular, to prove the exact integrability of the system [11]:

$$
\begin{aligned}
\partial\left(g_{0}^{-1} \partial g_{0} / \partial z_{-}\right) / \partial z_{+} & =\left[J_{-}, g_{0}^{-1} J_{+} g_{0}\right]+\left[\xi_{-}, g_{0}^{-1} \xi_{+} g_{0}\right], \\
\partial \xi_{ \pm} / \partial z_{\mp} & =\left[J_{ \pm}, g_{0}^{ \pm 1} \xi_{\mp} g_{0}^{\mp 1}\right] .
\end{aligned}
$$

The latter is related to the grading spectrum of the elements $A_{ \pm}\left(z_{+}, z_{-}\right)$in the subsuperspaces $\left(\mathfrak{G}_{0} \oplus \mathfrak{G}_{ \pm 2}\right)_{\overline{0}} \oplus\left(\mathfrak{G}_{ \pm 1}\right)_{\overline{1}}$ of the Lie superalgebra $\mathfrak{G}$, which is graded by means of the Cartan element $H$ of the subalgebra

$$
\operatorname{sl}(2):\left\{H, J_{ \pm} ;\left[H, J_{ \pm}\right]= \pm 2 J_{ \pm},\left[J_{+}, J_{-}\right]=H\right\}
$$

in $\left(\mathfrak{5}_{\overline{0}}\right.$. Here $g_{0}=\exp u\left(z_{+}, z_{-}\right) \in G_{0}(\Lambda)=\exp \left(\mathfrak{\xi}_{0}(\Lambda), u\left(z_{+}, z_{-}\right)\right.$, and $\xi_{ \pm}\left(z_{+}, z_{-}\right)$are the functions with the values in the even part of the Grassmann hull of $\mathfrak{G}_{0}$ and $\mathfrak{G}_{ \pm 1}$, respectively.

The exhaustive classification of various types of supermanifolds $V_{2 \mid 2}$ appearing requires certainly the description of all possible embeddings of the superalgebras $\operatorname{sl}(2)$ and $\operatorname{osp}(1,2)$ in $(\mathfrak{G}$.

\section{2.}

For the effective application of the algebraic approach in the investigation of the embeddings of two-dimensional manifolds $V_{2} \subset R_{N}$ supplied with the structure of Lie algebra $\left(5\right.$ the indicial system of Eqs. (A.2) with $n=2$ and $\bar{R}_{A B C D}=0$ (or its generalization in non-Riemannian case) should be reformulated in the form of representation (1.2) with the help of the explicit realization of the operators $A_{ \pm}$in the graded Lie algebra $(5$. Here the aforementioned arbitrariness in the choice of (pseudo-) normals to $V_{2}$ and the parameters on it is clearly related to transformation (1.3). Evidently it is necessary to realize the operators $A_{ \pm}$by the 
Cartan elements and the root vectors of 5 rather than with the help of the tensor basis (like $M_{\mathrm{AB}}$ ) to provide the possibility of considering the embeddings of manifolds supplied with the structure of various Lie algebras $(\mathfrak{b}$. Here we illustrate the afore-said by the example of the embeddings $V_{2} \subset R_{N}$, i.e. for $(\mathfrak{G} \cong O(N)$. Other types of simple Lie algebras can be considered independently or by the reduction over simple roots $\pi$ of $D_{\ell}$ or $B_{\ell}$, for example, $D_{4} \underset{\pi_{4}=\pi_{3}}{\longrightarrow} B_{3} \underset{\pi_{3}=\pi_{1}}{\longrightarrow} G_{2}$. For these algebras, as well as for the series $B_{\ell}$ and $D_{\ell}$, there is a complete Dynkin's classification of the embeddings of three-dimensional subalgebras. The study of the embeddings of non-Riemannian manifolds follows the completely analogical scheme and demands the use of non-compact real forms. In particular, nonRiemannian generalization with non-zero tensor $G_{(1)}^{A B}$ can be obtained in the basis of the algebra $\operatorname{SL}(N, \mathbb{R})$, with the symmetrical parts of the connections $\tilde{\gamma}_{C}^{\mathbb{A} B}$ (A.12) being conjugated to the symmetrical tangent matrices of this algebra.

Consider an embedding of the two-dimensional Riemannian manifold $V_{2}$ into the flat Euclidean enveloping space $R_{N}$ and realize its representation (1.2) with the help of the operators $A_{ \pm}=A_{ \pm}^{\mathrm{AB}}\left(z_{+}, z_{-}\right) M_{\mathrm{AB}}$, taking the values in the orthogonal Lie algebra of rank $[N / 2]$ with the elements $M_{\mathbb{A B}}=-M_{\mathbb{B A}}$ satisfying the commutation relations

$$
\left[M_{\mathbb{A B B}}, M_{\mathbb{C D}}\right]=\delta_{\mathrm{AC}} M_{\mathbb{B D}}+\delta_{\mathrm{IBD}} M_{\mathrm{AC}}-\delta_{\mathbb{I B C}} M_{\mathrm{AD}}-\delta_{\mathrm{AD}} M_{\mathbb{B C C}} .
$$

(Note the consideration of pseudo-Euclidean enveloping spaces is carried out quite analogically. The only difference being that the Kronecker symbols in (2.1) are supplied with the factors $\varepsilon_{\mathbf{A}}$. The latter express the pseudo-Euclidean character of the metric of $R_{N}$ and, correspondingly, the pseudo-orthogonality of the algebra.)

It would be convenient to come over to the complex structures

$$
q_{ \pm j}^{a} \equiv q_{j 1}^{a} \pm i q_{j 2}^{a}, \quad t_{ \pm}^{a b} \equiv t_{1}^{a b} \pm i t_{2}^{a b}, \quad \gamma_{ \pm}^{\mu \nu} \equiv \gamma_{1}^{\mu \nu} \pm i \gamma_{2}^{\mu \nu}, \quad e^{j \pm} \equiv e^{j 1} \pm i e^{j 2},
$$

in order to formulate the functions $A_{ \pm}^{\mathrm{ABB}}\left(z_{+}, z_{-}\right)$in terms of the coefficients of the fundamental forms of a manifold $V_{2}$ depending on the parameters $x_{j}=i^{j-1} z_{+}$ $+(-\mathrm{i})^{j-1} \mathrm{z}_{-}$. Then using relations $(2.1)$ one can easily verify by a direct check that the substitution of the operators $A_{ \pm}=A_{ \pm}^{\mathrm{AB}} \cdot M_{\mathrm{ABB}}$ with

$$
A_{ \pm}^{\mathbf{A B}}=\gamma_{ \pm}^{\mu v} \delta_{\mu}^{\mathbf{A}} \delta_{v}^{\mathbb{B}}+q_{ \pm j}^{a} e^{j \mu} \delta_{a}^{\mathbf{A}} \delta_{\mu}^{\mathbb{B}}+t_{ \pm}^{a b} \delta_{a}^{\mathbf{A}} \delta_{b}^{\mathbb{B}}
$$

in representation (1.2) leads to the Gauss, Peterson-Codazzi, and Ricci equations (A.2) for an embedding $V_{2} \subset R_{N}$. Here the $\partial / \partial x^{i}+(1 / 2) \gamma_{i}^{\mu \nu} M_{\mu \nu} \equiv \partial_{i}+\gamma_{i}^{21} M_{12}$ realizes the covariant differentiation of the corresponding tensor quantities. [Note the operator $\partial / \partial y^{A}+(1 / 2) \gamma_{A}^{\mathrm{AB}} \cdot M_{\mathrm{ABB}}$ plays the same role for the embeddings $V_{N} \subset R_{N+p}$ as the previous expression for $V_{2} \subset R_{N}$.]

The operators $A_{ \pm}$can be equivalently expanded over the elements $E^{A B}=-E^{B A}$,

$$
\left(E^{i j} \equiv e^{i \mu} e^{j v} M_{\mu \nu}, E^{i a} \equiv e^{i \mu} M_{\mu a}, E^{a b} \equiv M_{a b}\right),
$$

of the Lie algebra satisfying commutation relations (2.1), where the symbols $\delta_{\mathrm{ABB}}$ are replaced by

$$
\Delta^{A B} \equiv g^{i j} \delta_{i}^{A} \delta_{j}^{B}+\delta^{a b} \delta_{a}^{A} \delta_{b}^{B}
$$


Rewrite the expression for the operators $A_{ \pm}$in terms of the Cartan-Weyl invariant root basis. Due to the orthogonal algebras of even $(N=2 \ell)$ and odd $(N=2 \ell+1)$ dimensions corresponding to different classical series $\left(D_{\ell}\right.$ and $B_{\ell}$, respectively), consider first the even case following the notations of [12]. The series $D_{\ell}$ are described by the extended Dynkin diagram
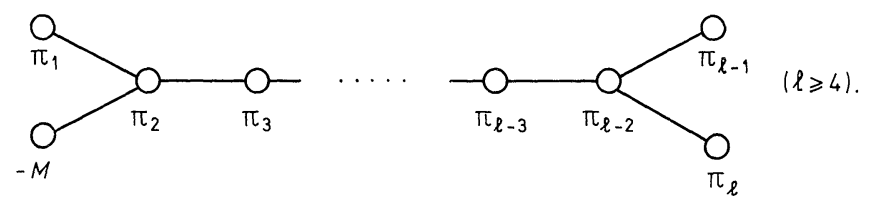

Here $-M=-\left(\pi_{1}+2 \sum_{\beta=2}^{\ell-2} \pi_{\beta}+\pi_{\ell-1}+\pi_{\ell}\right)$ is the minimal root, $\pi_{\alpha}$ are the simple roots. The Cartan generators $h_{\alpha}$ and the root vectors $X_{\alpha}^{ \pm}$corresponding to the simple roots satisfy the commutation relations

$$
\left[h_{\alpha}, h_{\beta}\right]=0, \quad\left[h_{\alpha}, X_{\beta}^{ \pm}\right]= \pm k_{\beta \alpha}^{\mathfrak{5}} X_{\beta}^{ \pm}, \quad\left[\mathrm{X}_{\alpha}^{+}, \mathrm{X}_{\beta}^{-}\right]=\delta_{\alpha \beta} \mathrm{h}_{\alpha},
$$

with $k^{\mathfrak{G}} \equiv k$ being the Cartan matrix of $\mathfrak{G}=D_{\ell}$. Denote the root vectors of all positive and negative roots of the algebra via $X_{\Lambda}^{ \pm}, 1 \leqq \Lambda \leqq \ell(\ell-1)$. Consider in the root space $\mathbb{R}^{\ell}$ the canonical basis $\left(\varepsilon_{\alpha}\right)$, where $\pi_{\alpha}=\varepsilon_{\alpha}-\varepsilon_{\alpha+1}, 1 \leqq \alpha \leqq \ell-1$, $\pi_{\ell}=\varepsilon_{\ell-1}+\varepsilon_{\ell}$.

In these notations the operators $A_{ \pm}$have the form

$$
A_{ \pm}=\sum_{\alpha=1}^{\ell} U_{ \pm}^{\alpha} h_{\alpha}+\sum_{1 \leqq \alpha<\beta \leqq \ell} \operatorname{Sp}\left(R_{ \pm}^{\alpha \beta} X_{\alpha \beta}\right)
$$

where the trace is calculated over the indices $i, j=1,2$ of the matrices

$$
\begin{aligned}
X_{\alpha \beta}^{i j} & \equiv\left\|\begin{array}{cc}
X_{\varepsilon_{\alpha}-\tilde{\varepsilon}_{\beta}}^{+} & X_{\varepsilon_{\alpha}+\tilde{\varepsilon}_{\beta}}^{-} \\
-X_{\varepsilon_{\alpha}+\tilde{\varepsilon}_{\beta}}^{+} & -X_{\varepsilon_{\alpha}}^{-}
\end{array}\right\|, \quad \tilde{\varepsilon}_{\beta} \equiv(-1)^{\ell \delta_{\beta} \varepsilon_{\beta}} ; \\
R_{ \pm i j}^{\alpha \beta} & \equiv v\left\|\begin{array}{cc}
r_{ \pm}^{2 \alpha-1,2 \beta-1} & r_{ \pm}^{2 \alpha-1,2 \beta} \\
r_{ \pm}^{2 \alpha, 2 \beta-1} & r_{ \pm}^{2 \alpha, 2 \beta}
\end{array}\right\| v^{-1}, \quad v \equiv\left\|\begin{array}{cc}
1 & i \\
1 & -i
\end{array}\right\| ; \\
U_{ \pm}^{\alpha} & \equiv-i\left[\begin{array}{c}
2 \sum_{\gamma=1}^{\alpha-1} t_{ \pm}^{2 \gamma+1,2 \gamma+2}+\gamma_{ \pm}^{21}
\end{array}\right], \quad 1 \leqq \alpha \leqq \ell-2 ; \\
U_{ \pm}^{\ell+v_{\ell}-1} & \equiv i\left[2 t_{ \pm}^{2 \ell-1,2 \ell}-\sum_{\gamma=1}^{\ell-1} t_{ \pm}^{2(\ell-\gamma)+1,2(\ell-\gamma)+2}-\frac{1}{2} \gamma_{ \pm}^{21}\right] ; \\
U_{ \pm}^{\ell-v_{\ell}} & \equiv-i\left[\sum_{\gamma=1}^{\ell-1} t_{ \pm}^{2(\ell-\gamma)+1,2(\ell-\gamma)+2}+\frac{1}{2} \gamma_{ \pm}^{21}\right] ; \quad v_{\ell} \equiv \begin{cases}1, & \text { odd } \ell \\
0, & \text { even } \ell .\end{cases}
\end{aligned}
$$

The functions $r_{ \pm}^{a A}=-r_{ \pm}^{A a}$ are

$$
r_{ \pm}^{\mu b} \equiv q_{ \pm j}^{b} e^{j \mu}, \quad r_{ \pm}^{a b} \equiv t_{ \pm}^{a b} ; \quad \mu=1,2 ; \quad 3 \leqq a, \quad b \leqq 2 \ell, \quad 1 \leqq A \leqq 2 \ell .
$$

Here the $3 d$ fundamental forms (A.4) are expressed via functions (2.7) or via matrices (2.6) as

$$
\begin{gathered}
Q_{3}^{a b}=r_{\theta_{1}}^{a A} \cdot r_{\theta_{2}}^{A b} d z_{\theta_{1}} d z_{\theta_{2}}=\left(v^{-1} R_{\theta_{1}}^{\alpha \gamma} R_{\theta_{2}}^{\gamma \beta} v\right)_{i j} d z_{\theta_{1}} d z_{\theta_{2}}, \\
\theta_{1}, \theta_{2} \equiv \pm ; \quad a \equiv 2 \alpha+i-2, \quad b \equiv 2 \beta+j-2 ; \quad i, j=1,2 .
\end{gathered}
$$


To single out the exactly integrable subclasses of system (A.2) resulting from the substitution of operators (2.5) in representation (1.2), consider, in accordance with the general construction of [6], the gradings of the algebra $D_{\ell}$ that are consistent with the integral embeddings of the three-dimensional subalgebra sl(2). Since Dynkin's procedure [9] for the description of all types of embeddings is uniform for all finite-dimensional simple Lie algebras $\mathfrak{G}$, we recall briefly the main steps of the procedure for arbitrary $\mathfrak{( \mathfrak { G }}$.

Each embedding is unambiguously defined (up to equivalence) by the decomposition structure of the Cartan element $H$ of the subalgebra sl(2) over the generators $h_{\alpha}$ of the algebra $\left(\mathfrak{5}, H=\sum_{\alpha=1}^{\ell} C_{\alpha} h_{\alpha}, \ell \equiv\right.$ rank $(\mathfrak{5}$. In their turn the unknown structure constants $C_{\alpha}$ of the embedding are determined by the following scheme. Consider the extended Dynkin diagram for $\mathfrak{G}$ and select its subset $P^{(s)} \equiv\left\{p_{1}, \ldots, p_{s} ; s \leqq \ell\right\}$ of any roots corresponding to some semisimple Lie

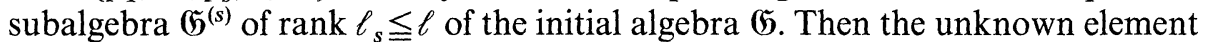
$H=\sum_{1 \leqq \alpha \leqq \ell_{s}} C_{\alpha} h_{p_{\alpha}}$ is found from the conditions $\left[H, X_{p_{\alpha}}^{ \pm}\right]= \pm 2 X_{p_{\alpha}}^{ \pm}$. Here the embedding of sl(2) in $\left(5\right.$, where $\left[H, X_{\alpha}^{ \pm}\right]= \pm 2 X_{\alpha}^{ \pm}, 1 \leqq \alpha \leqq \ell$, plays a peculiar role. The corresponding $3 d$-subalgebra is called the principal $3 d$-subalgebra. Really, the description of all embeddings $\operatorname{sl}(2) C(5$ is reduced to consideration of the principal $3 d$-subalgebras in all algebras $\left(\mathfrak{b}^{(s)}\right.$. (The exceptions are $\left[\frac{\ell-2}{2}\right]$ embeddings for the series $D_{\ell}$ and $\left[\frac{\ell^{\prime}-3}{2}\right]$ for $E_{\ell^{\prime}}, \ell^{\prime}=6,7,8$.) Therefore the structure of $H$ for other embeddings (non-principal) is established from this condition written in the form

$$
\left[H, X_{\alpha}^{ \pm}\right]= \pm 2 X_{\alpha}^{ \pm}, \quad 1 \leqq \alpha \neq s \leqq \ell ; \quad\left[H, X_{M}^{\mp}\right]= \pm 2 X_{M}^{\mp}
$$

for $1 \leqq s \leqq \ell$ and from the subsequent reduction. Formula (2.9) leads to

$$
\begin{gathered}
H=H^{(s)} \equiv \sum_{\alpha=1}^{\ell} k_{\alpha}^{(s)} h_{\alpha}, \quad k_{\alpha}^{(s)} \equiv 2 \sum_{\beta=1}^{\ell} k_{\alpha \beta}^{-1}-2 \eta_{s} k_{\alpha s}^{-1}, \\
\eta_{s} \equiv\left(1+\sum_{\beta=1}^{\ell} d_{\beta}^{M}\right) / d_{s}^{M},
\end{gathered}
$$

where $d_{\alpha}^{\Lambda}$ are the decomposition coefficients of the root $\Lambda$ over the simple roots, $\Lambda=\sum_{1 \leqq \alpha \leqq \ell} d_{\alpha}^{\Lambda} \pi_{\alpha}$. Thus, taking into account the relation

$$
\left[H, X_{A}^{ \pm}\right]= \pm 2\left(\sum_{1 \leqq \alpha \leqq \ell} d_{\alpha}^{\Lambda}-d_{s}^{\Lambda} \eta_{s}\right) X_{A}^{ \pm},
$$

we come to the following structure of the local part $\hat{\mathfrak{b}}$ of the algebra $\mathfrak{5}$ with the grading $H^{(s)}$ :

$$
\begin{aligned}
\mathfrak{G}_{0} & =\left\{h_{\alpha}, 1 \leqq \alpha \leqq \ell ; X_{\Lambda}^{+}, X_{\Lambda}^{-}: \sum_{\alpha=1}^{\ell} d_{\alpha}^{\Lambda}=d_{s}^{A} \eta_{s}\right\}, \\
\mathfrak{G}_{ \pm 1} & =\left\{X_{\Lambda}^{ \pm}: \sum_{\alpha=1}^{\ell} d_{\alpha}^{\Lambda}=d_{s}^{\Lambda} \eta_{s}+1\right\} ; \quad 1 \leqq s \leqq \ell .
\end{aligned}
$$


Here it seems natural that the case $s=0$ corresponds to the canonical grading [the principal subalgebra $\mathrm{sl}(2)$ of $[5]$, i.e.,

$$
\mathfrak{b}_{0}=\left\{h_{\alpha}, 1 \leqq \alpha \leqq \ell\right\}, \quad \mathfrak{b}_{ \pm 1}=\left\{X_{\alpha}^{ \pm}, 1 \leqq \alpha \leqq \ell\right\} .
$$

For the case of the series $D_{\ell}$ under consideration formulae $\left(2.11_{1}\right)$ lead to

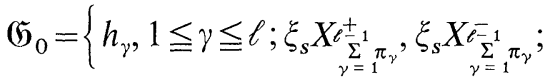

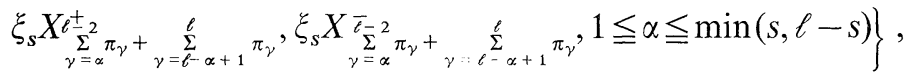

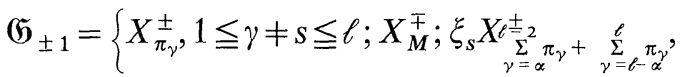

$$
\begin{aligned}
& 1 \leqq \alpha \leqq \min (s, \ell-s-1)\} \text {, } \\
& \xi_{s} \equiv \begin{cases}0, & s=1, \ell-1, \ell \\
1, & 2 \leqq s \leqq \ell-2\end{cases}
\end{aligned}
$$

or

$$
\begin{aligned}
\mathfrak{G}_{0}= & \left\{h_{\gamma}, 1 \leqq \gamma \leqq \ell ; \xi_{s} X_{\varepsilon_{1}-\varepsilon_{\ell}}^{ \pm} ; \xi_{s} X_{\varepsilon_{\alpha}+\varepsilon_{\ell-\alpha+1}}^{ \pm}, 1 \leqq \alpha \leqq \min (s, \ell-s)\right\} \\
\mathfrak{G}_{ \pm 1}= & \left\{X_{\varepsilon_{\gamma}-\varepsilon_{\gamma+1}}^{ \pm}, \gamma \neq s ; X_{\varepsilon_{\ell-1}+\varepsilon_{\ell}}^{ \pm} ; \xi_{s} X_{\varepsilon_{\alpha}+\varepsilon_{\ell-\alpha}}^{ \pm},\right. \\
& \left.1 \leqq \alpha \leqq \min (s, \ell-s-1) ; X_{\varepsilon_{1}+\varepsilon_{2}}^{\mp}\right\}
\end{aligned}
$$

As can be easily seen after excluding the corresponding set of points in scheme (2.3), some of these gradings are evidently equivalent.

Retain in expansion (2.5) only the terms with the elements of the subspaces $\mathfrak{b}_{0} \oplus \mathfrak{b}_{+1}$ and $\mathfrak{b}_{0} \oplus \mathfrak{b}_{-1}$ (for the operators $A_{+}$and $A_{-}$, respectively). Then taking into account formulas (2.12) we obtain the required relations for the quantities $r_{ \pm}^{a A}$ from (2.7). Let us enumerate all nonequivalent possibilities. For the case of even $\ell$ 's we have

$s=1$ :

$$
\begin{aligned}
& R_{+11}^{\alpha \beta}=R_{+11}^{\alpha \beta} \sum_{\gamma=2}^{\ell-1} \delta_{\alpha \gamma} \delta_{\beta \alpha+1}, \quad R_{+21}^{\alpha \beta}=R_{+21}^{\alpha \beta} \delta_{\alpha 1} \delta_{\beta 2}, \\
& R_{+12}^{\alpha \beta}=R_{+12}^{\alpha \beta} \delta_{\alpha \ell-1} \delta_{\beta \ell}, \quad R_{+22}^{\alpha \beta}=0 \\
2 \leqq S \leqq & {\left[\frac{\ell+1}{2}\right]: } \\
R_{+11}^{\alpha \beta}= & R_{+11}^{\alpha \beta}\left[\delta_{\alpha 1} \delta_{\beta \ell}+\sum_{\substack{\gamma=1 \\
\gamma \neq s}}^{\ell-1} \delta_{\alpha \gamma} \delta_{\beta \alpha+1}\right], \\
R_{+21}^{\alpha \beta}= & R_{+21}^{\alpha \beta}\left[\delta_{\alpha 1} \delta_{\beta 2}+\sum_{\gamma=1}^{s} \delta_{\alpha \gamma} \delta_{\beta \ell-\alpha+1}\right], \\
R_{+12}^{\alpha \beta}= & R_{+12}^{\alpha \beta}\left[\sum_{\gamma=1}^{s} \delta_{\alpha \gamma} \delta_{\beta \ell-\alpha+1}+\sum_{\gamma=1}^{\min (s, \ell-s-1)} \delta_{\alpha \gamma} \delta_{\beta \ell-\alpha}+\delta_{\alpha \ell-1} \delta_{\beta \ell}\right], \\
R_{+22}^{\alpha \beta}= & R_{+22}^{\alpha \beta} \delta_{\alpha 1} \delta_{\beta \ell} .
\end{aligned}
$$


According to the definition, the functions $R_{-i j}^{\alpha \beta}$ are constructed from $R_{+i j}^{\alpha \beta}$ via the formula

$$
R_{-i j}^{\alpha \beta}=\left[\left(R_{+}^{\alpha \beta}\right)^{-1} \operatorname{det} R_{+}^{\alpha \beta}\right]_{i j}^{*} .
$$

To consider the odd values of $\ell$ one has to make the replacement $R_{ \pm j 1}^{\alpha \ell} \rightleftarrows R_{ \pm j 2}^{\alpha \ell}$ in (2.13) and (2.14).

The solution of the same problem for the orthogonal algebras of odd dimensions is obtained by equating the simple roots $\pi_{\ell}$ and $\pi_{\ell-1}$ in the root system of $D_{\ell+1}$. This procedure corresponds to the transition from (2.3) to the $\pi$-system for the series $B_{\ell}$,

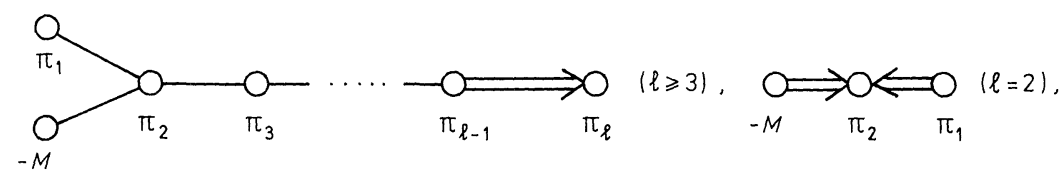

where

$$
-M \equiv-\pi_{1}-2 \sum_{2 \leqq \beta \leqq \ell} \pi_{\beta}
$$

or, in terms of the coefficient functions, to the equalities $r_{ \pm}^{2 \ell-1, A}=0$.

Thus, in accordance with expression (2.8), the classification of exactly integrable embeddings of $V_{2}$ into $R_{N}$ is defined by the structure of the $3 d$ fundamental forms with nonzero coefficient functions (2.13) and (2.14). It is via nonzero elements of the matrix $Q_{3}^{a b}$ that system (1.4) with $\xi_{ \pm}=0$ describing these embeddings can be formulated. For this to be accomplished, one has to reduce expansion (2.5) to the form [6]

$$
A_{+}=g_{0}^{-1} J_{+} g_{0}, \quad A_{-}=J_{-}+g_{0}^{-1} \partial g_{0} / \partial z_{-}
$$

by expressing the group parameters of the element $g_{0} \in \widetilde{G}_{0}$ through the coefficients at the differentials (A.4). Here the form-invariance of (1.2) with respect to transformation (1.3) with $\tilde{g}_{0} \in \widetilde{G}_{0}$ is applied. Then (2.16) leads to system (1.4) with $\xi_{ \pm}=0$, which allows [6] an explicit solution of the Goursat problem characterized by $2 \cdot \operatorname{dim}\left(\mathfrak{b}_{1}\right.$ arbitrary functions $\varphi_{+\alpha}\left(z_{+}\right)$and $\varphi_{-\alpha}\left(z_{-}\right), 1 \leqq \alpha \leqq \operatorname{dim} \mathfrak{G}_{1}$.

The present approach to the completely integrable systems is connected with a realization of the operators $A_{ \pm}$in the corresponding subspaces of the infinitedimensional Lie algebra $\widetilde{\mathfrak{G}}$ of finite growth [13]. Here the existence of finitedimensional representations of $\widetilde{\mathfrak{G}}$ provides the possibility of the nontrivial introduction of a spectral parameter and construction of the soliton-type solutions. In this situation the choice of a definite grading spectrum of the operators $A_{ \pm}$in $\widetilde{\mathfrak{G}}, A_{ \pm} \dot{\epsilon} \underset{0 \leqq m \leqq m_{ \pm}}{\bigoplus_{ \pm m}} \widetilde{\mathfrak{G}}_{ \pm m}$ is equivalent in a certain sense to their realization on the elements of the corresponding finite-dimensional Lie algebra $(\mathfrak{5}$, which form a degenerate (finite-dimensional) representation of the generators of $\widetilde{\mathfrak{G}}_{ \pm m}, 0 \leqq m \leqq m_{ \pm}$. 
3.

Consider supermanifold $V_{2 \mid 2}$ with the coordinates

$$
z^{a}=\left\{x^{i}, \theta^{\alpha} ; 1 \leqq i, \alpha \leqq 2\right\}
$$

embedded into the superspace $V_{N \mid M}$ with the coordinates

$$
Z^{\mathfrak{I}}=\left\{y^{A}, 1 \leqq A \leqq N ; \Theta^{\Omega}, 1 \leqq \Omega \leqq M\right\} ; \quad Z^{\mathfrak{A}}=Z^{\mathfrak{A}}(z)
$$

(For the basic notions of supermanifolds, see e.g. [14].) Introduce with the help of the components $E_{a}^{\mathfrak{Q}} \equiv \partial Z^{\mathfrak{A}} / \partial z^{a}$ of the repère, $\left(\partial / \partial z^{a}=E_{a}^{\mathfrak{Q}} \partial / \partial Z^{\mathfrak{A}}\right)$, the elements $\gamma_{a}(x ; \theta)$ $\equiv E_{a}^{\mathfrak{A}} \Gamma_{\mathfrak{O}}$ at the points $z$ in $V_{2 \mid 2}$, where $\Gamma_{\mathfrak{U}}(Z) \dot{\in} \mathfrak{G}(\Lambda)$ is a connection of $V_{N \mid M}$.

Then, if the components $\mathfrak{R}_{\mathfrak{Q B}}(Z ; \Gamma)$ are equal to zero, for $\gamma_{a}(z) \dot{\in} \mathfrak{\mathfrak { G }}(\Lambda)$ one has $\mathfrak{R}_{a b}(z ; \gamma)=0$.

It follows that up to the gauge transformation

$$
\gamma_{a} \rightarrow \tilde{g}_{0}^{-1} \gamma_{a} \tilde{g}_{0}+\tilde{g}_{0}^{-1} \partial \tilde{g}_{0} / \partial z^{a}, \quad \tilde{g}_{0} \in \exp \left(\tilde{b}_{0}(\Lambda),\right.
$$

the even elements $\gamma_{i}(z)$ from $\mathfrak{G}(\Lambda)$ completely define the odd $\gamma_{\alpha}(z)$. Hence, it is sufficient to consider the relation $\mathfrak{R}_{12}\left(x ; \gamma_{i}(x ; \theta)\right)=0$.

For convenience we represent it in a component form by using the expansion

$$
\gamma_{i}(x ; \theta)=\gamma_{i}^{(0)}(x)+i \bar{\theta} \gamma_{i}^{(1)}(x)+\frac{1}{2} \bar{\theta} \theta \gamma_{i}^{(2)}(x) \text {. }
$$

Here $\gamma_{i}^{(0)}$ and $\gamma_{i}^{(2)}$ are scalar functions taking values in $\mathbf{5}$, and $\gamma_{i}^{(1)} \equiv\left\{\gamma_{i}^{\mu}\right\}$ are the twocomponent columns of Majorana spinors in $\left(\mathfrak{5} ; \bar{\theta} \equiv\left(-\theta_{-}, \theta_{+}\right), \theta \equiv\left(\begin{array}{l}\theta_{+} \\ \theta_{-}\end{array}\right)\right.$. Because of the recurrent structure of the equations for $\gamma_{i}^{(s)}, 0 \leqq s \leqq 2$, they are defined by the solution $\gamma_{i}^{(0)}(x)=g^{-1} \partial g / \partial x^{i}$ of the equation $\mathfrak{R}_{12}\left(x ; \gamma_{i}^{(0)}(x)\right)=0$, as well as by the arbitrary spinor $\left\{\varphi^{\mu}(x)\right\}$ and the scalar $f(x)$ taking values in $(\mathfrak{G}$. The latter are related to $\gamma_{i}^{(s)}$ by the formulas

$$
\gamma_{j}^{\mu}(x)=g^{-1} \partial \varphi^{\mu}(x) / \partial x^{j} g, \quad \gamma_{j}^{(2)}(x)=g^{-1}\left[\partial f(x) / \partial x^{j}-\bar{\varphi} \partial \varphi / \partial x^{j}\right] g,
$$

where $g(x)$ is a function with the values in the even part of the Grassmann hull of the supergroup $G$ with the superalgebra $\mathbb{6}$.

Thus, the system $\mathfrak{R}_{a b}(z ; \gamma)=0$ is reduced to Eq. (1.2),

$$
z_{ \pm} \equiv 1 / 2\left(x^{1} \mp i x^{2}\right), \text { with } A_{ \pm} \equiv \gamma_{1}^{(0)}(x) \pm i \gamma_{2}^{(0)}(x)
$$

being the even elements of the Grassmann hull of the superalgebra 65 . [Here the coefficients $a_{ \pm}^{\kappa}\left(z_{+}, z_{-}\right)$at even $F_{\kappa}\left(\in \mathfrak{G}_{\overline{0}}\right)$ in $A_{ \pm}$are scalar functions, whereas those at the odd ones $\left(\in \mathfrak{G}_{\overline{1}}\right)$ are anticommuting elements of the Grassmann algebra.] As has already been said, in order to single out the exactly integrable subclass of equations for $a_{ \pm}^{\kappa}$, one should define a grading spectrum of $A_{ \pm}$.

The completely integrable supermanifolds are also contained in this construction and are described by the equations [in particular of form (1.4)] associated, in accordance with [6], with infinite-dimensional Lie superalgebras $\overrightarrow{\mathfrak{G}}$ of finite growth [15]. The enveloping superspace is still supplied with the structure of the finite-dimensional Lie superalgebra $\mathbf{6 5}$, which realizes here the degenerate representation of $\tilde{\mathfrak{F}}$. The elements $A_{ \pm}$have a corresponding spectrum over $\widetilde{\mathfrak{G}}$, but 
not with respect to $\mathfrak{G}$. This consideration essentially differs from an analogous one in the conventional space. We shall explain it in the following example.

It is well-known that in the case of the embedding of $V_{2}$ into $R_{3}$, the integrable surfaces are described both by the Liouville equation (L) related to the algebra $A_{1}(\mathrm{sl}(2))$, and the completely integrable Sine-Gordon equation (SG), corresponding to the infinite-dimensional simple Lie algebra $\tilde{A}_{1}$ of finite growth (see Appendix B). Here we have a minimal possible realization of $\tilde{A}_{1}$ in the basis $\operatorname{sl}(2)$ for both equations due to the presence of the degenerate representation of $\tilde{A}_{1}$ in sl(2). But in the case of the integrable supersurfaces $V_{212}$, corresponding to the supersymmetry L-equations [finite-dimensional superalgebra osp $(1,2)$ ] and to the SG-one [infinite-dimensional superalgebra $C^{(2)}(2)$ of finite growth], this situation does not occur. (Note that these equations (as in [16]) follow from (1.4) with

$$
\begin{gathered}
\mathrm{L}: g_{0}=\exp \left(-\frac{1}{2} \varrho \mathscr{H}\right), \quad J_{ \pm}= \pm \frac{1}{2}\left(\mathscr{Y}_{ \pm}\right)^{2}, \quad \xi_{ \pm}=\left(\begin{array}{c}
1 \\
-i
\end{array}\right) \omega^{ \pm} \mathscr{Y}_{ \pm} ; \\
\mathrm{SG}: g_{0}=\exp \left(-\frac{1}{2} \varrho \mathscr{H}_{1}\right), \quad J_{ \pm}=\frac{1}{2 \sqrt{2} i}\left(\mathscr{Y}_{ \pm 1}-\mathscr{Y}_{ \pm 2}\right)^{2}, \\
\xi_{ \pm}=\frac{i}{\sqrt{2 m}}\left(\begin{array}{c}
1 \\
-i
\end{array}\right) \omega^{ \pm}\left(\mathscr{Y}_{ \pm 1}+\mathscr{Y}_{ \pm 2}\right) .
\end{gathered}
$$

Here $\left\{\mathscr{H} ; \mathscr{Y}_{ \pm}\right\}$and $\left\{\mathscr{H}_{1}\left(=-\mathscr{H}_{2}+\mathscr{C}\right) ; \mathscr{Y}_{ \pm i}\right\}$ are the canonical generators of the basis of the subsuperspaces $\left\{\mathfrak{5}_{0} ; \mathfrak{5}_{ \pm 1}\right\}$ of the superalgebras osp $(1,2)$ and $C^{(2)}(2)$, respectively; $\varrho\left(z_{+}, z_{-}\right)$is a scalar function, $\omega^{ \pm}\left(z_{+}, z_{-}\right)$are components of a Majorana spinor with anticommuting values.)

The minimal possibility of their uniform description is connected with the consideration of the superspace supplied with a structure of the finite-dimensional superalgebra $\operatorname{sl}(1,2)$ with the scheme $\otimes-\otimes$. Through its generators $\mathfrak{b}_{0}=\left\{h_{i}\right.$; $i=1,2\}$ and $\mathfrak{G}_{ \pm 1}=\left\{Y_{i}^{ \pm} ; i=1,2\right\}$ the basis of the subsuperspaces $\widetilde{\mathfrak{G}}_{0}$ and $\widetilde{\mathfrak{G}}_{ \pm 1}$ of $C^{(2)}(2)$ in the degenerate representation is expressed by the relations

$$
\mathscr{Y}_{1}^{ \pm}=\sqrt{2} \sum_{i} c_{i}^{ \pm 1} Y_{i}^{ \pm}, \mathscr{Y}_{2}^{ \pm}=\mp \sqrt{2} \sum_{i} d_{i}^{ \pm 1} Y_{i}^{\mp}, \quad \mathscr{H}_{1}=-\mathscr{H}_{2}=2\left(h_{1}+h_{2}\right),
$$

where $c_{i}$ and $d_{i}$ are non-zero constants, and $\sum_{i} c_{i} d_{i}=0$. One may also choose $\mathscr{Y}_{1}^{ \pm}$ and $\mathscr{H}_{1}$ of the indicated form as the corresponding generators of osp $(1,2)$.

\section{4.}

As is known, every finite-dimensional simple Lie algebra $\mathfrak{G}$ over $\mathbb{C}$ in the canonical grading may be associated with the corresponding two-dimensional Toda lattice with the required invariance properties. Its random superalgebraic generalization, which is equivalent to the replacement [in (1.2) with $A_{ \pm} \dot{\epsilon}(5]$ of $\partial / \partial z_{ \pm}$by the supersymmetric covariant derivatives $\mathscr{D}_{ \pm}= \pm \partial / \partial \theta_{\mp}+i \theta_{\mp} \partial / \partial z_{ \pm}$, and, correspondingly, of $A_{ \pm}$by odd elements spanned over the root vectors of the simple roots of a contragradient Lie superalgebra (for the latter see [17]). It is not 
always that this generalization turns out to be supersymmetric. [In particular, under the condition of a positively defined kinetic energy of bosonic components this only holds for the superalgebras osp $(1,2)$ and $C^{(2)}(2)$ [18]; see also [19].]

The above consideration shows that the construction of supersymmetric equations, describing the integrable supermanifolds $V_{2 \mid 2}$, is not possible for all superalgebras and/or their gradings. For a supersymmetric generalization of the two-dimensional Toda lattice one should describe the minimal (in the sense of [6]) embedding of osp $(1,2)$ into the contragradient Lie superalgebras $(\mathfrak{5}$, which contain them. As was shown by D. A. Leites, these are, in particular, the superalgebras $\mathrm{sl}(n, n+1)$. For them Dynkin's scheme $\otimes-\cdots-\infty-\otimes$ is associated with the subsuperspaces

$$
\begin{gathered}
\mathfrak{b}_{0}=\left\{h_{i} ; 1 \leqq i \leqq 2 n\right\}, \quad \mathfrak{G}_{ \pm 1}=\left\{Y_{i}^{ \pm} ; 1 \leqq i \leqq 2 n\right\}, \\
\mathfrak{G}_{ \pm 2}=\left\{X_{\alpha}^{ \pm} \equiv\left[Y_{\alpha}^{ \pm}, Y_{\alpha+1}^{ \pm}\right] ; 1 \leqq \alpha \leqq 2 n-1\right\} .
\end{gathered}
$$

The component form of the arising supersymmetric equations:

$$
\mathscr{D}_{+} \mathscr{D}_{-} \varphi_{i}=\exp (k \varphi)_{i},
$$

coincides with system (1.4), with

$$
g_{0}=\exp \sum_{i=1}^{2 n} h_{i} \varrho_{i}\left(z_{+}, z_{-}\right) ; \quad J_{ \pm}=\sum_{\alpha=1}^{2 n-1} X_{\alpha}^{ \pm} ; \quad \xi_{ \pm}=\sum_{i=1}^{2 n} Y_{i}^{ \pm} \omega_{i}^{ \pm} .
$$

Here $(k \varphi)_{i} \equiv \sum_{j=1}^{2 n} k_{i j} \varphi_{j}$,

$$
\varphi \equiv\left\{\varphi_{i}\left(z_{+}, z_{-}\right)=\varrho_{i}+i \bar{\theta}\left(k^{-1} \omega\right)_{i}+\frac{1}{2} \bar{\theta} \theta \exp (k \varrho)_{i}\right\}
$$

is the multiplet of bosonic superfields and $k$ is the Cartan matrix of

$$
\operatorname{sl}(n, n+1) ; \quad k_{i j}=\delta_{i j+1}+\delta_{i+1 j}, \quad 1 \leqq i, j \leqq 2 n .
$$

\section{Appendix A}

In this appendix following the classical monograph by Eisenhart [2] we briefly give the necessary information from the theory of Riemannian manifolds as well as certain definitions of non-Riemannian geometry $[3,4]$.

Consider the Riemannian space $V_{N}$ of an arbitrary dimension $N$ with coordinates $y^{A}$ and metric $G_{A B}(y)=G_{B A}$ (which is generally indefinite) consistent with the symmetric connection $\Gamma_{B C}^{A}$. An embedding of the Riemannian manifold $V_{n}$ with coordinates $x^{i}$ into $V_{N}$ is determined by an analytical dependence $y^{A}=f^{A}\left(x^{i}\right)$ with matrix $\partial f^{A} / \partial x^{i} \equiv y_{, i}^{A}$ of rank $n$. Here ${ }^{3}$

$$
\begin{gathered}
G_{A B} E_{C}^{A} E_{D}^{B}=\eta_{C D}, \quad \eta^{C D} E_{C}^{A} E_{D}^{B}=G^{A B}, \\
E_{C}^{A} \equiv Y_{, i}^{A} \delta_{C}^{i}+n_{a}^{A} \delta_{C}^{a} ; \quad \eta_{C D} \equiv g_{i j} \delta_{C}^{i} \delta_{D}^{j}+\varepsilon_{a} \delta_{a b} \delta_{C}^{a} \delta_{D}^{b},
\end{gathered}
$$

3 From now on the summation convention is used for all types of indices: $i, j, k, \ell(\mu, v, \varrho, \tau)$ $=1, \ldots, n ; A, B, C, D(\mathbb{A}, \mathbb{B}, \mathbb{C}, \mathbb{D})=1, \ldots, N ; a, b, c, d=n+1, \ldots, N ; \Xi, \Lambda, \Omega, \Psi=N+1, \ldots, N+p ;$ $\mathfrak{A}, \mathfrak{B}, \mathfrak{C}, \mathfrak{D}=1, \ldots, N+p ; p \geqq 0$ 
where $n_{a}^{A}$ are components of the vector fields in $V_{N}$ normal to $V_{n}$ at its points, $g_{i j}(x)$ is the metric in $V_{n}$; the numbers $\varepsilon_{a}$ are equal to +1 or -1 . Then $V_{n}$ is described by its fundamental tensors $g_{i j}(x)=g_{j i}, q_{i j}^{a}(x)=q_{j i}^{a}, t_{i}^{a b}(x)=-t_{i}^{b a}$ with $q_{i j}^{a}$ and $t_{i}^{a b}$ being the components of the second quadratic forms and torsion vectors, respectively. These functions satisfy the Gauss, Peterson-Codazzi, and Ricci equations,

$$
\begin{gathered}
R_{i j k \ell}=\varepsilon_{a}\left(q_{i k}^{a} q_{j \ell}^{a}-q_{i \ell}^{a} q_{j k}^{a}\right)+\bar{R}_{A B C D} y_{, i}^{A} y_{, j}^{B} y_{, k}^{C} y_{, \ell}^{D} ; \\
q_{i j ; k}^{a}-q_{i k ; j}^{a}=\varepsilon_{b}\left(t_{k}^{b a} q_{i j}^{b}-t_{j}^{b a} q_{i k}^{b}\right)+\bar{R}_{A B C D} y_{, i}^{A} n_{a}^{B} y_{, j}^{C} y_{, k}^{D} ; \\
t_{j ; k}^{a b}-t_{k, j}^{a b}+\varepsilon_{c}\left(t_{j}^{c a} t_{k}^{b b}-t_{k}^{c a} t_{j}^{c b}\right)+g^{\ell m}\left(q_{l j}^{a} q_{m k}^{b}-q_{l k}^{a} q_{m j}^{b}\right) \\
+G^{A E} \bar{R}_{E B C D} n_{A}^{a} n^{B b} y_{, j}^{C} y_{, k}^{D}=0 .
\end{gathered}
$$

Here $\bar{R}_{A B C D}$ is the Riemannian curvature tensor for the metric $G_{A B}$ defined at the points of $V_{n}$; semicolon denotes covariant differentiation with respect to the metric $g_{i j}$. The equations represent the integrability conditions of the Gauss-Weingarten derivative formulae for the vectors $y_{, i}^{A}$ and $n_{a}^{A}$ in the moving frame,

$$
\begin{aligned}
& y_{; i j}^{A}=\varepsilon_{a} q_{i j}^{a} n_{a}^{A}-\bar{\Gamma}_{B C}^{A} y_{, i}^{B} y_{, j}^{C} ; \\
& n_{a ; i}^{A}=-q_{i j}^{a} g^{j k} y_{, k}^{A}+\varepsilon_{b} b_{i}^{b a} n_{b}^{A}-\bar{\Gamma}_{B C}^{A} y_{, i}^{C} n_{a}^{B} .
\end{aligned}
$$

(In what follows the factors $\varepsilon_{a}$ will be omitted for the sake of brevity.) It is obvious that the corresponding equations for manifolds supplied with a structure of an arbitrary Lie group (not only orthogonal) can be written down analogously.

In the classical theory of surfaces apart from the first and second fundamental quadratic forms

$$
\begin{gathered}
Q_{1} \equiv G_{A B} \cdot D y^{A} \cdot D y^{B}=g_{i j} d x^{i} d x^{j}, \\
Q_{2}^{a} \equiv-G_{A B} \cdot D y^{A} \cdot D n_{a}^{B}=q_{i j}^{a} d x^{i} d x^{j} \\
D y^{A} \equiv d y^{A}=y_{, i}^{A} d x^{i}, \quad D n_{a}^{A} \equiv n_{a ; i}^{A} d x^{i},
\end{gathered}
$$

the third fundamental form

$$
Q_{3}^{a b} \equiv G_{A B} D n_{a}^{A} D n_{b}^{B}=\left(q_{i k}^{a} g^{k \ell} q_{l j}^{b}-t_{i}^{a c} t_{j}^{c b}\right) d x^{i} d x^{j},
$$

is sometimes applied. Similar to the two forms mentioned above the other one also allows the direct geometrical interpretation and plays an important role in what follows.

Equations (A.2) and (A.3) are very much simplified for the embeddings of the manifold $V_{n}$ into a flat space $R_{N}, N \geqq n+p$ ( $p$ is a class index of $V_{n}$ ). They can be completely formulated in terms of $V_{n}$ and naturally do not contain the terms with the Riemannian tensor and the connection of $V_{N}$. For this reason it often appears to be convenient to consider the triple of spaces $V_{n} \subset V_{N} \subset R_{N+p}$ for the investigation of properties of the embeddings of $V_{n}$ into the Riemannian enveloping space $V_{N}$ (not necessarily flat) and for the solution of their classification problem ${ }^{4}$. The calculations for the investigation of the embeddings $\left(V_{2} \subset V_{N}\right.$, in particular) are provided by the following formulae on the base of the triple $V_{n} \subset V_{N} \subset R_{N+p}$. Let us

4 Apparently in algebraic terms the transition from $V_{N}$ to $R_{N}$ is equivalent to a contraction of the corresponding algebra 
introduce the repére $E_{\mathbf{A}}^{A}(y)$ in an enveloping space $V_{N}$,

$$
E_{\mathrm{A}}^{A} E^{\mathrm{A} B}=G^{A B}, \quad G_{A B} E_{\mathrm{A}}^{A} E_{\mathrm{BB}}^{B}=\delta_{\mathrm{A} \mathbb{B}},
$$

and thereby construct the "spin connections" $\gamma_{A}^{\mathrm{AB}}=-\gamma_{A}^{\mathrm{BA} A}$,

$$
\partial E_{A}^{\mathrm{A}} / \partial y^{B}+\gamma_{B}^{\mathrm{A} \mathrm{B}} E_{\mathrm{BB} A}-\bar{\Gamma}_{A B}^{C} E_{C}^{\mathrm{A}}=0 .
$$

It is easy to show that the Riemannian tensor in $V_{N}$ is expressed via these connections

$$
\partial \gamma_{D}^{\mathrm{AB}} / \partial y^{C}-\partial \gamma_{C}^{\mathrm{ABB}} / \partial y^{D}-\gamma_{D}^{\mathrm{A} \mathbb{C}_{\gamma}} \gamma_{C}^{\mathbb{C B B}}+\gamma_{C}^{\mathrm{AAC}} \gamma_{D}^{\mathbb{C B B}}=\bar{R}_{A B C D} E^{\mathrm{A} A} E^{\mathbb{B B} B}
$$

Then the embeddings of $V_{n}$ into $V_{N}$ allow the following reduction:

$$
y_{, i}^{D} \gamma_{D}^{\mathrm{ABB}}=\gamma_{i}^{\mu \nu} \delta_{\mu}^{\mathrm{A}} \delta_{v}^{\mathrm{B}}-q_{i j}^{a} e^{j \mu} \delta_{a}^{\mathrm{A}} \delta_{\mu}^{\mathrm{B}}+t_{i}^{a b} \delta_{a}^{\mathrm{A}} \delta_{b}^{\mathbb{B}},
$$

which is equivalent to the derivative formulae (A.3) for $V_{n} \subset V_{N}$ due to (A.6). Here $\gamma_{i}^{\mu \nu}(x)$ and $e_{\mu}^{i}(x)$ are analogous to $\gamma_{C}^{\mathbf{A} B \mathbb{B}}$ and $E_{\mathbb{A}}^{A}$ quantities, respectively, defined in $V_{n}$.

In the case of non-Riemannian spaces $V_{n} \subset V_{N}$ the derivative formulae for the vectors $y_{, i}^{A}$ and pseudo-normals $n_{a}^{A}$ are essentially complicated, as well as the integrability equations resulting from them. The latter generalize Eqs. (A.2). They contain three types of new tensors

$$
\omega_{i j}^{a} \equiv n_{A, B}^{a} y_{, i}^{A} y_{, j}^{B} ; \quad \ell_{a j}^{i} \equiv n_{a, B}^{A} x_{, A}^{i} y_{, j}^{B} ; \quad \ell_{a i}^{b} \equiv n_{a, B}^{A} n_{A}^{b} y_{, i}^{B} ;
$$

and are expressed by

$$
\begin{aligned}
& y_{; i j}^{A}=-\omega_{i j}^{a} n_{a}^{A}-\bar{L}_{B C}^{A} y_{, i}^{B} y_{, j}^{G} ; \\
& n_{a, i}^{A}=\ell_{a i}^{j} y_{, j}^{A}+\ell_{a i}^{b} n_{b}^{A}-\bar{L}_{B C}^{A} y_{, i}^{C} n_{a}^{B} ;
\end{aligned}
$$

where the symbol ";" denotes the covariant differentiation with respect to the connection $L_{l j}^{k}$ in $V_{n} ; \bar{L}_{B C}^{A}$ is the connection in $V_{N}$, both being asymmetrical, generally speaking. When the determinant of $\omega_{i j}^{a}$ is not equal to zero and the pseudo-normals can be chosen in such a way that $\ell_{a i}^{b}=0$, the tensors $g^{i j}$ (nondegenerate with $\left.\operatorname{det} \ell_{a k}^{i} \neq 0\right)$ and $g_{i j}$ are defined from the relations $g^{i j} \omega_{j k}^{a}=\ell_{a k}^{i}, g_{i j} g^{j k}=\delta_{i}^{k}, g^{i j} g_{j k}=\delta_{k}^{i}$. In this context the tensors $g_{i j}$ and $g^{i j}$ are used only for raising and lowering the indices of the tensor quantities of $V_{n}$. Here a variant with $\bar{L}_{B C D}^{A} \equiv \bar{L}_{B D, C}^{A}-\bar{L}_{B C, D}^{A}$ $+\bar{L}_{B D}^{E} \bar{L}_{E C}^{A}-\bar{L}_{B C}^{E} \bar{L}_{E D}^{A}=0$ represents an analogue of a flat enveloping space of Riemannian geometry. In what follows we shall call, in brief, the spaces $V_{N}$ with zero $\bar{R}_{A B C D}$ flat. In terms of the tensor $G^{A B}$ and connections $L_{B C}^{A}$ and $L_{B C}^{\prime A}$ realizing the covariant differentiation of co- and contravariant tensors, respectively, the Weyl and Schouten generalizations of Riemannian spaces can be expressed by the introduction of three tensors in $V_{N}$

$$
G_{; C}^{A B} \equiv G_{(1) C}^{A B}, \quad L_{B C}^{A}-L_{C B}^{A} \equiv G_{(2) B C}^{A}, \quad L_{B C}^{A}-L_{B C}^{\prime A} \equiv G_{(3) B C}^{A},
$$

equal to zero in the Riemannian case. Here, defining in $V_{N}$ the repére and spin connections by formulae (A.5) and (A.6), one gets convinced that the symmetrical parts of these connections are expressed by the formula

$$
\tilde{\gamma}_{C}^{\mathrm{A} \mathbb{B}}+\tilde{\gamma}_{C}^{\mathrm{BA}}=\tilde{E}_{D}^{\mathrm{BB}} \tilde{E}_{E}^{\mathrm{A}} G_{(1) C}^{D E} .
$$




\section{Appendix B}

Let us illustrate the general conclusions by the examples of non-trivial embeddings of $V_{2}$ into the enveloping spaces $R_{3}$ and $R_{5}$ related to the algebras $B_{1}\left(\simeq A_{1}\right)$ and $B_{2}$ and then trace the reduction to the case of the enveloping space supplied with the structure of the algebra $G_{2}$.

$B_{1}$ : For this case formula (2.5) is reduced to the form

$$
A_{ \pm}=u^{ \pm} H+f_{+}^{ \pm} X^{+}+f_{-}^{ \pm} X^{-} \text {, }
$$

where $f_{+}^{ \pm} \equiv q_{ \pm j} e^{j-}, f_{-}^{ \pm} \equiv q_{ \pm j} e^{j+}, u^{ \pm} \equiv(i / 2) \gamma_{ \pm}^{12}$. (Here and in what follows the inessential normalizing factors at the root vectors will be omitted for the sake of brevity.) The condition that the coefficients at $X^{-}$in $A_{+}$and at $X^{+}$in $A_{-}$be equal to zero, i.e. $f_{-}^{+}=f_{+}^{-}=0$, leads to

$$
q_{1 j} e^{j 1}-q_{2 j} e^{j 2}=0, \quad q_{1 j} e^{j 2}+q_{2 j} e^{j 1}=0 .
$$

Hence, $q_{i k} g^{k j}=q_{i j}^{-1} \operatorname{det}(q \cdot e)$. In the invariant form this condition is written as

$$
q_{i k} g^{k \ell} q_{\ell j}=\delta_{i j} \cdot \exp \varrho \quad \text { or } \quad Q_{3}=\exp \varrho \cdot\left(d x^{j}\right)^{2} .
$$

Putting (B.1) with $f_{-}^{+}=f_{+}^{-}=0$ into representation (1.2), we come to the system of equations

$$
u_{, z_{-}}^{+}-u_{, z_{+}}^{-}=f_{+}^{+} f_{-}^{-}, \quad\left(\ln f_{+}^{+}\right)_{, z_{-}}=-2 u^{-}, \quad\left(\ln f_{-}^{-}\right)_{, z_{+}}=2 u^{+} .
$$

As a consequence of the latter the function $\varrho$ satisfies the Liouville equation $\varrho_{, z_{+} z_{-}}=2 \exp \varrho$. With the conformally-flat metric, $g_{k \ell}=\lambda^{-2} \delta_{k \ell} \exp \varrho, \lambda=$ const, condition (B.2) in terms of the tensor $b_{i}^{j}=b_{j}^{i}=q_{i k} g^{k j}$ takes the form $b_{i}^{k} b_{j}^{k}=\lambda^{2} \delta_{i j}$. Then it follows that the exactly integrable embedding described by the Liouville equation corresponds either to the minimal surface with $\sum_{j} b_{j}^{j}=0$ or to the constant curvature surface with $b_{i}^{j}=\lambda \delta_{i}^{j}$.

For the Tchebycheff metric the constant curvature surface is well known to be described by the (completely integrable) Sine-Gordon equation $\varrho_{z_{z_{+}}}=2 \exp \varrho$ $-2 \exp (-\varrho)$. To obtain this equation in the framework of the given construction conditions $f_{+}^{+} f_{-}^{+}=\lambda, f_{-}^{-} f_{+}^{-}=\lambda^{-1}$. The latter correspond to the canonical grading of the infinite-dimensional Lie algebra $\tilde{A}_{1}$ of finite growth [13] in its degenerated representation (with $\tilde{X}_{1}^{ \pm}=X^{ \pm}, \tilde{X}_{2}^{ \pm}=\lambda^{ \pm 1} X^{\mp} ; \tilde{h}_{1}=-\tilde{h}_{2}=H$ ) and they are equivalent to

$$
q_{i k} g^{k \ell} q_{\ell j}=\delta_{i j}\left[\delta_{i 1} \exp \varrho+\delta_{i 2}(\exp \varrho-1)\right]
$$

or

$$
Q_{3}=\exp \varrho \cdot\left(d x^{1}\right)^{2}+(\exp \varrho-1) \cdot\left(d x^{2}\right)^{2}
$$

$B_{2}$ : Here the operators $A_{ \pm}$have the form

$$
\begin{aligned}
A_{ \pm}= & u_{1}^{ \pm} h_{1}+u_{2}^{ \pm} h_{2}+f_{+1}^{ \pm} X_{1}^{+}+f_{-1}^{ \pm} X_{1}^{-}+f_{+2}^{ \pm} X_{2}^{+}+f_{-2}^{ \pm} X_{2}^{-} \\
& +f_{+12}^{ \pm} X_{12}^{+}+f_{-12}^{ \pm} X_{12}^{-}+f_{+122}^{ \pm} X_{122}^{+}+f_{-122}^{ \pm} X_{122}^{-},
\end{aligned}
$$


where

$$
\begin{aligned}
& f_{1}^{ \pm}=\left(q_{ \pm j}^{3}+i q_{ \pm j}^{4}\right) e^{j-}, \quad f_{-1}^{ \pm}=\left(q_{ \pm j}^{3}-i q_{ \pm j}^{4}\right) e^{j+} ; \\
& f_{+122}^{ \pm}=\left(q_{ \pm j}^{3}-i q_{ \pm j}^{4}\right) e^{j-}, \quad f_{-122}^{ \pm}=\left(q_{ \pm j}^{3}+i q_{ \pm j}^{4}\right) e^{j+} ; \\
& f_{+2}^{ \pm}=t_{ \pm}^{35}+i t_{ \pm}^{45}, \quad f_{-2}^{ \pm}=t_{ \pm}^{35}-i t_{ \pm}^{45} ; \\
& f_{+12}^{ \pm}=q_{ \pm j}^{5} e^{j-}, \quad f_{ \pm 12}^{ \pm}=q_{ \pm j}^{5} e^{j+} ; \quad u_{1}^{ \pm}=-2 \gamma_{ \pm}^{12}, \quad u_{2}^{ \pm}=\gamma_{ \pm}^{12}-t_{ \pm}^{34} .
\end{aligned}
$$

The indices at the root vectors denote the number of the corresponding simple roots $\pi_{1}$ and $\pi_{2}$ over which the given root is expanded. For the case under consideration we obtain two nonequivalent gradings consistent with the integral embeddings of $A_{1}$ in $B_{2}$. The local parts for these gradings are written (up to the Weyl transformations) as

$$
\begin{array}{cc}
\mathfrak{G}_{0}=\left\{h_{1}, h_{2}\right\}, \quad \mathfrak{G}_{ \pm 1}=\left\{X_{1}^{ \pm}, X_{2}^{ \pm}\right\} ; \\
\mathfrak{G}_{0}=\left\{h_{1}, h_{2} ; X_{2}^{+}, X_{2}^{-}\right\}, \quad \mathfrak{G}_{ \pm 1}=\left\{X_{1}^{ \pm}, X_{12}^{ \pm}, X_{122}^{ \pm}\right\} .
\end{array}
$$

In the former case corresponding to the exactly integrable system (the Toda lattice for $B_{2}$ )

$$
\varrho_{\alpha, z_{+} z_{-}}=k_{\alpha \beta} \exp \varrho_{\beta}, \quad k=\left\|\begin{array}{rr}
2 & -2 \\
-1 & 2
\end{array}\right\|,
$$

the conditions for the grading spectrum of $A_{ \pm}$,

$$
f_{+122}^{ \pm}=f_{-122}^{ \pm}=f_{+12}^{ \pm}=f_{-12}^{ \pm}=f_{+1}^{-}=f_{+2}^{-}=f_{-1}^{+}=f_{-2}^{+}=0,
$$

lead to

$$
\begin{gathered}
q_{i k}^{3} g^{k \ell} q_{\ell j}^{3}=\delta_{i j} \exp \varrho_{1}=q_{i k}^{4} g^{k \ell} q_{\ell j}^{4} ; \\
q_{i k}^{3} g^{k \ell} q_{\ell j}^{4}=\varepsilon_{i j} \exp \varrho_{1} ; \quad q_{i k}^{a} g^{k \ell} q_{\ell j}^{5}=0 \quad \forall a=3,4,5 ; \\
t_{i}^{35}=-\varepsilon_{i j} t_{j}^{45}, \quad\left(\left(t_{1}^{35}\right)^{2}+\left(t_{2}^{35}\right)^{2}=\exp \varrho_{2}\right) ; \quad \varepsilon \equiv\left\|\begin{array}{rr}
0 & 1 \\
-1 & 0
\end{array}\right\| .
\end{gathered}
$$

In the latter case corresponding to the string-type exactly integrable system [20],

$$
\begin{gathered}
\varrho_{1, z_{+} z_{-}}=2 \exp \varrho_{1}-2 \frac{\sinh \left[\left(\varrho_{1}-\varrho_{2}\right) / 2\right]}{\cosh ^{3}\left[\left(\varrho_{1}-\varrho_{2}\right) / 2\right]} \omega_{, z_{+}} \omega_{, z_{-}}, \\
\varrho_{2, z_{+} z_{-}}=2 \exp \varrho_{2}+2 \frac{\sinh \left[\left(\varrho_{1}-\varrho_{2}\right) / 2\right]}{\cosh ^{3}\left[\left(\varrho_{1}-\varrho_{2}\right) / 2\right]} \omega_{, z_{+}} \omega_{, z_{-}}, \\
\left\{\tanh ^{2}\left[\left(\varrho_{1}-\varrho_{2}\right) / 2\right] \omega_{, z_{+}}\right\}_{z_{-}}+\left\{\tanh ^{2}\left[\left(\varrho_{1}-\varrho_{2}\right) / 2\right] \omega_{, z_{-}}\right\}_{, z_{+}}=0,
\end{gathered}
$$

the conditions for the grading spectrum of $A_{ \pm}$,

$$
f_{-1}^{+}=f_{+1}^{-}=f_{-12}^{+}=f_{+12}^{-}=f_{-122}^{+}=f_{+122}^{-}=0,
$$

lead to

$$
q_{i k}^{a} g^{k \ell} q_{\ell j}^{a}=\delta_{i j} \exp \left[\varrho_{(a)}\left(z_{+}, z_{-}\right)\right]
$$


Here the functions $\varrho_{(a)}\left(z_{+}, z_{-}\right)$are related to the solutions of the string-type equations given above.

$G_{2}$ : The operators $A_{ \pm}$realizing the embedding of 2-dimensional surface into the flat space supplied with the structure of the group $G_{2}$ can be constructed with the corresponding operators for $V_{2} \subset R_{7}$ by equating the roots in $\pi$-system of $B_{3}$ $\left(\pi_{3}=\pi_{1}\right)$. This procedure is equivalent to the conditions

$$
\begin{aligned}
\left(q_{ \pm j}^{a}+i \theta q_{ \pm j}^{a+1}\right) e^{j(-\theta)}=t_{ \pm}^{9-a, 7}+i \theta t_{ \pm}^{8-a, 7} ; & a=3,5 ; \quad \theta= \pm ; \\
i \theta q_{ \pm j}^{7} e^{j(-\theta)}=t_{ \pm}^{35}-t_{ \pm}^{46}-i \theta\left(t_{ \pm}^{36}+t_{ \pm}^{45}\right) ; & t_{ \pm}^{34}+t_{ \pm}^{56}=\frac{1}{2} \gamma_{ \pm}^{21}
\end{aligned}
$$

and leads to the following expression

$$
\begin{aligned}
A_{ \pm}= & -i \gamma_{ \pm}^{21} h_{1}-i\left(\gamma_{ \pm}^{21}+2 t_{ \pm}^{34}\right) h_{2}+\left(q_{ \pm j}^{3}-i \theta q_{ \pm j}^{4}\right) e^{j \theta} X_{1}^{\theta} \\
& +\left(q_{ \pm j}^{5}-i \theta q_{ \pm j}^{6}\right) e^{j \theta} X_{12}^{\theta}-\left(q_{ \pm j}^{3}+i q_{ \pm j}^{4}\right) e^{j \theta} X_{11122}^{\theta} \\
& -\left(q_{ \pm j}^{5}+i \theta q_{ \pm j}^{6}\right) e^{j \theta} X_{1112}^{\theta}+\left[t_{ \pm}^{35}+t_{ \pm}^{46}-i \theta\left(t_{ \pm}^{36}-t_{ \pm}^{45}\right)\right] X_{2}^{\theta} \\
& +\left[t_{ \pm}^{35}-t_{ \pm}^{46}+i \theta\left(t_{ \pm}^{36}+t_{ \pm}^{45}\right)\right] X_{112}^{\theta} .
\end{aligned}
$$

[Here we use the same notations as in formula (B.4) for the indices of the simple roots of $G_{2}$.] The subsequent singling out of exactly integrable embeddings follows completely the general scheme described above.

Acknowledgements. The author gratefully thanks A. T. Fomenko, D. A. Leites, A. N. Leznov, Yu. I. Manin, and A. V. Razumov for fruitful discussions.

\section{References}

1. Saveliev, M.V.: Classification problem for exactly integrable embeddings of two-dimensional manifolds and coefficients of the third fundamental forms. Report at the workshop on nonlinear and turbulent processes in physics. (Kiev, October, 1983)

2. Eisenhart, L.P.: Riemannian Geometry. Princeton, NJ: University Press 1926

3. Eisenhart, L.P.: Non-Riemannian geometry. Am. Math. Soc. Colloquium Publ., Vol. VIII, 1927

4. Kagan, V.F.: Riemann's geometric ideas and their modern development. Moscow, 1933 (in Russian)

5. Rashewsky, P.K.: Riemannian geometry and the tensor analysis. Moscow: Nauka 1964 (in Russian)

Dubrovin, B.A., Novikov, S.P., Fomenko, A.T.: Modern geometry. Moscow: Nauka 1979 (in Russian)

Kobayashi, S., Nomizu, K.: Foundations of differential geometry. New York: Interscience 1963

Helgason, S.: Differential geometry, Lie groups, and symmetric spaces. New York: Academic Press 1978

6. Leznov, A.N., Saveliev, M.V.: Nonlinear equations and graded Lie algebras. Sov. Prob. Mat. Mat. Anal. 22 (1984) (in Russian)

Representation theory and integration of nonlinear spherically symmetric equations to gauge theories. Commun. Math. Phys. 74, 111-118 (1980)

Two-dimensional exactly and completely integrable systems. Commun. Math. Phys. 89, 59-75 (1983)

7. Leznov, A.N., Shabat, A.B., Smirnov, V.G.: Internal symmetry group and integrability conditions for two-dimensional dynamical systems. Sov. J. Theor. Math. Phys. 51, 10-18 (1982) (in Russian)

Shabat, A.B., Yamilov, R.I.: Exponential systems. Preprint UFA. 23 (1981) (in Russian) 
8. Gabeskiria, M.A., Saveliev, M.V.: Lax-type representation for embeddings of Riemannian manifold. Preprint IHEP 83-58, Serpukhov (1983)

9. Dynkin, E.B.: Semisimple subalgebras of semisimple Lie algebras. Mat. Sb. 30, 349-462 (1952) (in Russian)

Maximal subgroups of the classical groups. Trudi MMO 1 (1952) (in Russian)

10. Geometrical ideas in physics. Manin, Yu.I. (ed.). Moscow: Mir 1983 (in Russian)

11. Leznov, A.N.: Exactly integrable two-dimensional dynamical systems related with supersymmetric algebras. Preprint IHEP 83-7, Serpukhov (1983) (in Russian)

12. Bourbaki, N.: Groupes et algebres de Lie. Paris: Herman 1968

13. Kac, V.G.: Simple irreducible graded Lie algebras of finite growth. Math. USSR - Izv. 2, 1271-1311 (1968) (in Russian)

14. Kostant, B.: Graded manifolds, graded Lie theory, and prequantization. In: Lecture Notes in Mathematics, Vol. 570, pp. 177-306. Berlin, Heidelberg, New York: Springer 1977

Berezin, F.A.: Introduction in algebra and analysis with anticommuting variables. Moscow: MGU (1983) (in Russian)

Leites, D.A.: Theory of supermanifolds. Petrozavodsk (1983) (in Russian)

15. Kac, V.G.: Adv. Math. 30, 85-136 (1978)

Leites, D.A., Serganova, V.V., Feigin, B.L.: Kac-Moody superalgebras. Report at the Intern. Seminar on Group theoretical methods in physics (Zvenigorod, November, 1982). Moscow: Nauka 1983 (in Russian)

16. Chaichian, M., Kulish, P.P.: On the method of inverse scattering problem and Bäcklund transformations for supersymmetry equations. Phys. Lett. B 78, 413-416 (1978)

17. Kac, V.G.: Lie superalgebras. Adv. Math. 26, 8-96 (1977)

18. Olshanetsky, M.A.: Supersymmetric two-dimensional Toda lattice. Commun. Math. Phys. 88, 63-76 (1983)

19. Kirillova, R.Ya.: Explicit solutions for generalized Toda lattices related to classical Lie superalgebras. Trudi LOMI 123, 98-111 (1983) (in Russian)

20. Barbashov, B.M., Nesterenko, V.V., Cherviakov, A.M.: General solutions of nonlinear equations in the geometric theory of the relativistic string. Commun. Math. Phys. 84, 471-486 (1982)

Communicated by Ya. G. Sinai

Received January 18, 1984; in revised form March 6, 1984

Note added in proof. Consider an arbitrary finite-dimensional Lie superalgebra $\mathfrak{g}$, which is graded by means of the Cartan element $H$ of its osp $(1,2)$ subsuperalgebra with the basis $H, Y_{ \pm}$, $J_{ \pm}=Y_{ \pm}^{2}\left(\left[H, Y_{ \pm}\right]_{-}= \pm Y_{ \pm},\left[Y_{+}, Y_{-}\right]_{+}=H\right)$. Then the construction given in Sects. 3 and 4 leads to the following system of supersymmetric equations

$$
\mathscr{D}_{+}\left(g^{-1} \mathscr{D}_{-} g\right)=\left[Y_{-}, g^{-1} Y_{+} g\right]_{+}
$$

with Eqs. (4.2) as a particular case. Here $g\left(z_{ \pm}, \theta_{ \pm}\right)$is the function of the Grassmann hull of the group $G_{0}$ with the algebra $\mathfrak{g}_{0}$. This result has been recently obtained in our paper with Prof. A. N. Leznov "Two-Dimensional Supersymmetric Nonlinear Equations Associated with the Embeddings of the Superalgebra osp $(1,2)$ into the Lie Superalgebras" (Preprint IHEP 84-59, Serpukhov, 1984; to appear in Sov. J. Theor. Math. Phys.). 